\title{
The controllable synthesis of the hybrid silica monolithic materials with the stable skeleton macroporous structure
}

\author{
Guo-Chuang Cao, Si-Ying Wang, Yun-kai Lvªnd Xin-Ying Qin
}

Corresponding author: Tel: 13633321313; E-mail: Ivyunkai@126.com. (Yun-kai Lv)

Present address: College of Chemistry and Environmental Science, Hebei University, Baoding 071002, China.

Keywords: Hybrid monolithic column, sole precursor, formation mechanism controlled microchannel

ABSTRACT. A new hybrid monolithic column (HMC) was prepared in stainless steel chromatographic column using nitric acid as catalysis and methyltrimethoxysilane as sole precursor. The influences of the precursor ratio, catalyst concentrations, gelation temperatures on properties of HMC were investigated, and the formation mechanism of the HMC was discussed. The obtained monolithic column was characterized by (SEM) and chromatographic evaluation. The results showed that the homogeneous network skeleton structure was formed in the HMC with a controlled microchannel $(10.3 \mu \mathrm{m})$ and nanopore $(13.6 \mathrm{~nm})$ and the lower shrinking ratio of HMC in comparison with silica monolithic column were obtained.

\section{Introduction}

The macroporous monolithic materials have used in various fields for heterogeneous catalysis and separation ${ }^{[1]}$. The monolithic materials include polymer materials ${ }^{[2]}$, inorganic materials ${ }^{[3]}$ and organic-inorganic hybrid materials ${ }^{[4]}$. The hybrid column research is mainly focused on capillary monolithic column ${ }^{[5]}$. There are few reports on the application of stainless steel monolithic columns.

The traditional stainless steel monolithic column has the advantages of high hardness, strong toughness, large internal diameter, uniform structure, and large column capacity. Lv's groups reported an internal surface reversed-phase monolithic column was prepared by in situ modification of hybrid monolithic columns (HMC) in a stainless steel column ${ }^{[6]}$. The HMC was synthesized via the cocondensation of methyltrimethoxysilane (MTMS) and tetraethoxysilane (TEOS). The synthetic conditions were optimized for obtaining the uniform microchannel and the stable skeleton structure.

In this work, organic-silica hybrid monolithic columns were prepared by sol-gel process in the stainless steel column with methyltrimethoxysilane as the sole precursor. The effect of reaction conditions on the properties of the HMC was studied, and the formation mechanism of the HMC was investigated. The morphology, porosity, and stability of the HMC were characterized by scanning electron microscopy and chromatographic analysis.

\section{Materials and Methods}

\section{Instrumental and materials}

Methyltrimethoxysilane (MTMS) are obtained from Nanjing Lianye Chemical Co. Ltd. (Shanghai, China). All other chemicals are of analytical or HPLC grade and used. Double deionized water (DDW) is used in all the experiment. All sample solutions for HPLC are filtered through a $0.22 \mu \mathrm{m}$ filter to remove large polymers and particles.

A Shimadzu LC-20A HPLC system (Kyoto, Japan) was used in this study, and a Venusil XBP C18 column $(5 \mu \mathrm{m}, 250 \mathrm{~mm} \times 4.6 \mathrm{~mm}$, Agela, China) was used as column pressure contrast experiment.

\section{Methods}

Hybrid monolithic columns were prepared in stainless steel column $(150 \mathrm{~mm} \times 4.6 \mathrm{~mm}) .2 \mathrm{~mL}$ of MTMS, $0.75 \mathrm{~mL}$ of methanol and $0.5 \mathrm{~mL}$ of $1.0 \mathrm{~mL} \mathrm{~L}^{-1}$ nitric acid were vortexed in an Erlenmeyer flask. The mixture was sonicated for $10 \mathrm{~min}$ and then poured into a stainless steel 
column sealed at the bottom and top. Then, the stainless steel column was placed vertically in a water bath at $40{ }^{\circ} \mathrm{C}$ for $24 \mathrm{~h}$. The monolithic column was eluted with $\mathrm{MeOH}$ for removing the unreacted residues and then dried at $40{ }^{\circ} \mathrm{C}$ for $12 \mathrm{~h}$. The reaction conditions that change during the conditional optimization process will be described in detail below.

\section{Results and discussion}

\section{The influence of methanol amount on the skeleton structure}

In this experiment, the different amount of methanol was added to the reaction mixture. Methanol was used as reaction solvent because the rather apolar precursor would initially not dissolve in the aqueous phase ${ }^{[7]}$. On the other hand, the methanol acts as porogen for the formation of an appropriate pore structure ${ }^{[8]}$. As shown in Table I, while the amount of methanol increased, the pore diameter and the skeleton diameter increased.

Table I The influence of methanol amount on the pore and skeleton structure

\begin{tabular}{cccccc}
\hline Column & $\begin{array}{c}\text { MTMS } \\
(\mathrm{mL})\end{array}$ & $\begin{array}{c}\mathrm{MeOH} \\
(\mathrm{mL})\end{array}$ & $\begin{array}{c}\mathrm{HNO}_{3} \\
\left(\mathrm{~mol} \mathrm{~L}^{-1}\right)\end{array}$ & $\begin{array}{c}\text { Through-pore size } \\
(\mu \mathrm{m})\end{array}$ & $\begin{array}{c}\text { Skeleton size } \\
(\mu \mathrm{m})\end{array}$ \\
\hline HMC-a & 2 & 0.55 & 1.0 & 4.3 & 2.2 \\
HMC-b & 2 & 0.65 & 1.0 & 10.3 & 4.3 \\
HMC-c & 2 & 0.75 & 1.0 & 13.2 & 4.5 \\
HMC-d & 2 & 0.85 & 1.0 & 19.3 & 6.4 \\
HMC-e & 2 & 0.95 & 1.0 & 23.7 & 10.3 \\
HMC-f & 2 & 1.05 & 1.0 & macroscopic phase separation \\
\hline
\end{tabular}

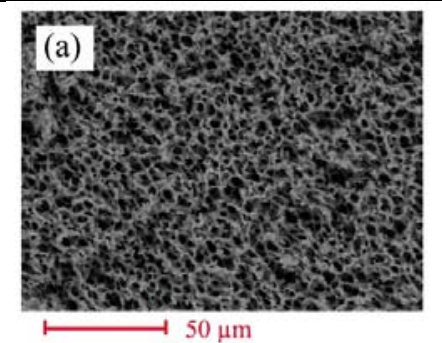
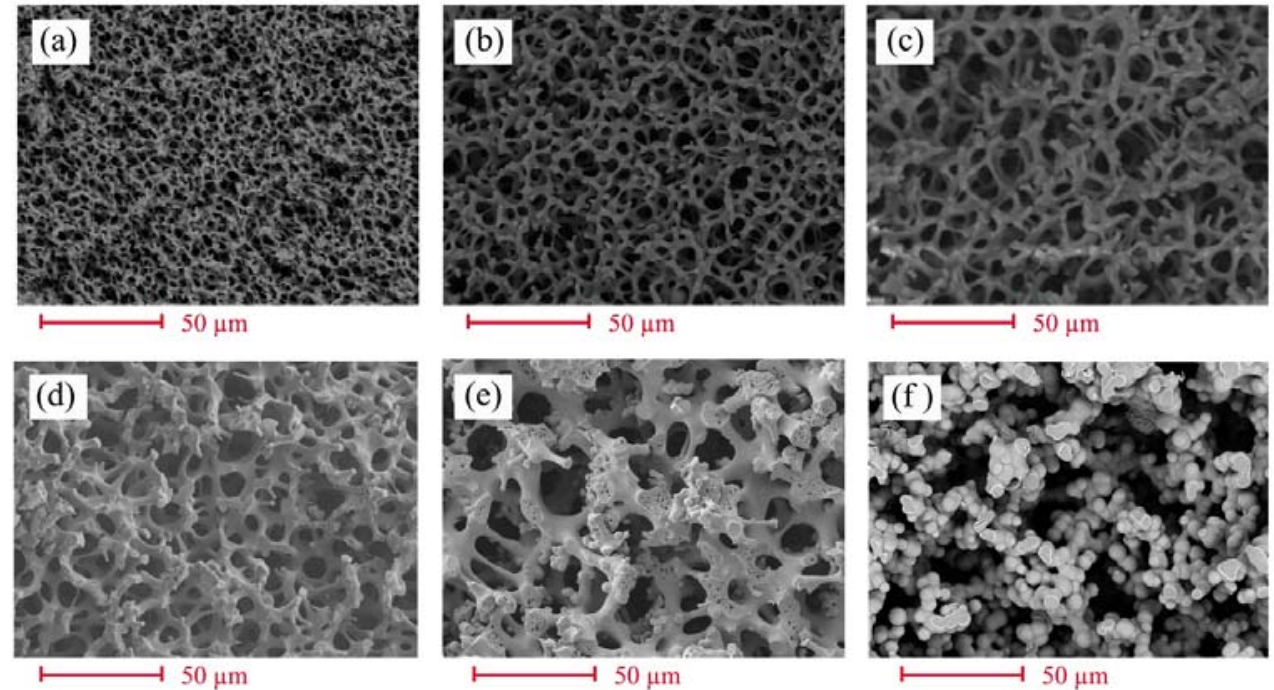

Figure 1. SEM images of HMC at different methanol amount. (a) $0.55 \mathrm{ml}$; (b) $0.65 \mathrm{ml}$; (c) $0.75 \mathrm{ml}$; (d) $0.85 \mathrm{ml}$; (e) $0.95 \mathrm{ml}$; (f) $1.05 \mathrm{ml}$.

The SEM images of the monolithic column were shown in Figure 1(a-f) at different amount of methanol. This effect is caused by macroscopic phase separation. With the increasing of pore diameter and skeleton diameter, the skeleton structure of monolithic column gradually changed from network structure to closed pore structure and particle accumulation structure. A higher fraction of methanol in the reaction mixture results in a higher dilution of hydrolysed precursor molecules and thus in a slowing-down of the polycondensation kinetics. The phase separation mechanism is also affected by the higher dilution as the point of saturation of the solvent with oligo and polymers is reached later. The effect on the phase separation seems to be smaller than the effect on the condensation as the increase of pore and skeleton diameters at higher contents of methanol in the initial reaction mixture indicates. 


\section{The influence of catalyst concentration}

Table II The influence of the nitric acid concentration on the pore and skeleton structure

\begin{tabular}{cccccc}
\hline Column & $\begin{array}{c}\text { MTMS } \\
(\mathrm{mL})\end{array}$ & $\begin{array}{c}\mathrm{MeOH} \\
(\mathrm{mL})\end{array}$ & $\begin{array}{c}\mathrm{HNO}_{3} \\
\left(\mathrm{~mol} \mathrm{~L}^{-1}\right)\end{array}$ & $\begin{array}{c}\text { Through-pore size } \\
(\mu \mathrm{m})\end{array}$ & $\begin{array}{c}\text { Skeleton size } \\
(\mu \mathrm{m})\end{array}$ \\
\hline HMC-C1 & 2 & 0.75 & 0.6 & 1.7 & 0.8 \\
HMC-C2 & 2 & 0.75 & 0.7 & 2.4 & 1.0 \\
HMC-C3 & 2 & 0.75 & 0.8 & 5.3 & 2.5 \\
HMC-C4 & 2 & 0.75 & 0.9 & 10.3 & 4.3 \\
HMC-C5 & 2 & 0.75 & 1.0 & 13.2 & 4.5 \\
HMC-C6 & 2 & 0.75 & 1.1 & 18.2 & 6.1 \\
\hline
\end{tabular}

The influence of the concentration of nitric acid as catalyst on the morphology of the synthesized monolithic material was investigated, the results were listed in Table II. As the concentration of nitric acid increased, the intensity of the reaction increased and the phase separation tendency increased, and the pore size and the skeleton diameter increased. Under the conditions, the hydrolysis rate should increase with increasing concentration of catalyst. The same should be valid for the polycondensation reaction. The results obtained here seem to indicate that indeed the phase separation starts earlier with increasing the catalyst concentration.

\section{Stability of monolithic columns}

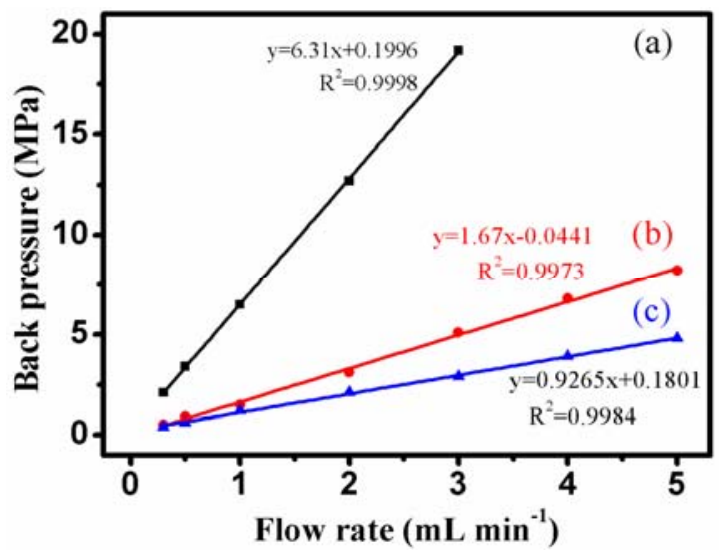

Figure 2. The influence of different flow rates on back pressures of columns at different mobile phases. Mobile phase: (a) MeOH (C18 column); (b) THF (HMC); (c) MeOH (HMC)

When methanol and tetrahydrofuran (THF) were used as the mobile phase, the linear relationship between flow rate and back pressure is good. As can be seen from Figure 2, the correlation coefficients are 0.9984 and 0.9973 , respectively. When the column pressure reached 8 $\mathrm{MPa}$, the pore structure remained good stability, high permeability. Compared with C18 column, the column pressure increase of the monolithic column is obviously smaller, which is suitable for the rapid separation and analysis.

\section{Conclusion}

The sol-gel method was successfully employed for the production of monolithic materials in format with MTMS as sole precursor. The organic-silica hybrid monolithic column with was prepared in-situ in a stainless steel column. By the inspection of several conditions such as, the percentage of MTMS and catalyst of nitric acid concentration, we found that the change of the reaction conditions can affect the transformation of sol-gel and decomposition process, thus affecting the pore diameter of product properties. Scanning the electronic microscopy shows that the monolithic column has a uniform skeleton network structure, the mesoporous pore size can reaches to $13.6 \mathrm{~nm}$. Under the optimum reaction conditions, we have created a double holes and uniform skeleton structure of organic silicone hybrid monolithic column, the column has a strong mechanical stability. 


\section{Acknowledgment}

This work was supported by the National Natural Science Foundation of China (No. 21375032), the Natural Science Foundation of Hebei Province (No. B2016201213, B2016201210).

\section{References}

[1] Thomas L. Recent developments in high-performance liquid chromatography stationary phases[J]. Anal. Chem., 2013, 85 (2): 579-589

[2] Frantisek S. Porous polymer monoliths: Amazingly wide variety of techniques enabling their preparation[J]. J. Chromatogr. A, 2010, 1217 (6): 902-924

[3] Walsh Z., Paull B., Macka M. Inorganic monoliths in separation science: A review[J]. Anal. Chim. Acta, 2012, 750:28-47

[4] Zhu T., Row K. H. Preparation and applications of hybrid organic-inorganic monoliths: A review[J]. J. Sep. Sci., 2012, 35 (10-11):1294-1302

[5] Wu R., Hu L., Wang F., et al. Recent development of monolithic stationary phases with emphasis on microscale chromatographic separation[J]. J. Chromatogr. A, 2008, 1184: 369-392.

[6] Lv Y.-K., Yang L., Liu X.-H., et al. Preparation and evaluation of a novel molecularly imprinted hybrid composite monolithic column for on-line solid-phase extraction coupled with HPLC to detect trace fluoroquinolone residues in milk[J]. Anal. Methods, 2013, 5: 1848-1855.

[7] Laschober S., Sulyok M., Rosenberg E. Tailoring the macroporous structure of monolithic silicabased capillary columns with potential for liquid chromatography[J]. J. Chromatogr. A, 2007, 1144: 55-62.

[8] Kaji H., Nakanishi K., Soga N. Formation of porous gel morphology by phase separation in gelling alkoxy-derived silica. Affinity between silica polymers and solvent[J]. J. Non-cryst. Solids, 1995, 181: 16-26. 